\title{
NECESSIDADES E EXPECTATIVAS DE ATUAÇÃO DO PSICÓLOGO EM CIRURGIA E PROCEDIMENTOS INVASIVOS
}

\section{NEEDS AND EXPECTATIONS OF PSYCHOLOGIST INTERVENTION IN SURGERY ANDINVASIVE PROCEDURES}

\author{
Tereza Cristina Cavalcanti Ferreira de ARAUJO' \\ Elaine Lima Machado ARRAES ${ }^{2}$
}

\begin{abstract}
RESUMO
Do ponto de vista histórico, a inserção do psicólogo na área hospitalar se deu primordialmente por intervenções focais ou sistemáticas junto a pacientes submetidos a procedimentos cirúrgicos ou invasivos em serviços de saúde. Todavia, apesar da continuidade e relevância dessas atividades, é possível constatar a escassez de pesquisas sobre a eficácia dos métodos de preparação psicológica.Assim sendo, este trabalho propõe,inicialmente, uma revisão crítica da literatura, para em seguida, apresentar um levantamento realizado em unidades hospitalares, envolvendo observações do atendimento e entrevistas com profissionais da saúde. As necessidades e expectativas relativas à atuação do psicólogo são analisadas e discutidas, concluindo-se pela importância da formação e treinamento desse profissional. Os autores sugerem ainda a realização de maiores investigações, especialmente de metaanálise de estudos em contexto brasileiro. Para tanto, recomendam levantamentos das intervenções mais adotadas, a exemplo da presente iniciativa.
\end{abstract}

Palavras-chave: métodos de preparação psicológica, cirurgia, procedimentos invasivos, atuação do psicólogo.

\begin{abstract}
In historicalterms, the psychologistinsertion intohospitalsetting has primordially been through focal and methodical interventions with patients who were submitted to surgical or invasive procedures at health services. However, despite the continuity and importance of these activities, it is possible to confirm the scarcity of researches about efficacy of psychological preparationmethods. In that case, this study initially proposes a critical review of literature, and
\end{abstract}

\footnotetext{
(1) Doutora pela Universidade de Paris X - Nanterre. Professora do Laboratório de Saúde e Desenvolvimento Humano. Instituto de Psicologia - Universidade de Brasília

(2) Bacharel em Psicologia pela Universidade de Brasília. Endereço para correspondência: UnB - Colina - Bloco G - Ap. 205 - Brasília - DF - 70910-900 - E-mail: araujotc@ unb.br
} 
afterwards it presents a survey realized in hospital units which includes observations of medical attention and interviews with health professionals. The needs and expectations related to psychologist intervention are analyzed and discussed. The results show the importance of the formation and training of such professional. The authors also suggest the realization of more investigations specially into meta-analysis study in the Brazilian context. Therefore, they recommend of surveys of the more utilized interventions, like this study.

Key-words: psychological preparation methods, surgery, invasive procedures, psychologist intervention.

\section{INTRODUÇÃO}

Historicamente, os primeiros trabalhos voltados para os aspectos psicológicos da experiência cirúrgica diziam respeito a relatos descritivos apresentados por médicos que se defrontavam com dificuldades no acompanhamento de pacientes, nos quais identificavam distúrbios psiquiátricos relacionados a intervenção operatória (Bainbridge, 1930; Macgraw, 1930; Cannon, 1935; Ebaugh, 1937, conforme citados em Janis, 1974). Naquela época, Freud (1920) já salientava que a investigação sobre as reações psíquicas a ameaças externas constituía um tema promissor para as ciências, tendo inclusive abordado estudos de casos, nos quais destacava a importância da atividade lúdica para a elaboração de vivências desta natureza. No entanto, em seu trabalho sobre a fobia do Pequeno Hans, publicado primeiramente em 1909 e reeditado em diversas ocasiões, Freud menciona, mas não analisa, as implicações psicológicas da amigdalectomia praticada no menino aos dois anos e meio de idade (Aberastury, 1972). Adotando a perspectiva psicanalítica, Sophie Morgenstern, em 1937, estabeleceu associação entre o mutismo de um menino de oito anos e uma extração de amígdalas (Aberastury, 1972). HélèneDeutsch, em 1942, também apresentava algumas observações psicanalíticas sobre cirurgia (Janis, 1974). Mas, as primeiras tentativas documentadas de intervir efetivamente para reduzir ansiedade e desordens psíquicas em indivíduos submetidos a procedimentos médicos invasivos datam da década de quarenta. Assim, Shirley e Poyntz (1941) desenvolviam atividades distrativas com crianças ansiosas em situação de procedimentos médicos, chamando a atenção para os distúrbios comportamentais decorrentes da separação entre mãe e filho no ambiente hospitalar.

Desde então, diversas técnicas preparatórias foram desenvolvidas e até programas foram implementados em instituições de saúde. Todavia, de modo geral, apenas a prática clínica tem norteado a adoção de estratégias de preparação psicológica, o que conduz ao uso indiscriminado dos métodos ou à escolha de técnicas baseada exclusivamente em preferências pessoais dos profissionais. Ora, somente a partir da compreensão dos mecanismos e variáveis envolvidos em tais intervenções, é possível determinar e indicar o formato preparatório ideal (Reading, 1979; Saile, Burgmeier \& Schmidt, 1988). Este, aliás,é o interesse central das pesquisas sobre o tema, as quais têm utilizado o recurso da comparação entre as diversas técnicas, a fim de possibilitar uma melhor compreensão da eficácia de cada uma, em função do tipo de clientela e do contexto de aplicação. Vale destacar que desde agosto de 1998, a Portaria 3.432 do Ministério da Saúde estabelece para o credenciamento de unidades de tratamento intensivo, a existência de um serviço de psicologia. Portanto, o presente trabalho tem por objetivo realizar uma revisão da literatura especializada, abordando criticamente as principais variáveis estudadas, no intuito 
de fundamentar a análise e a discussão sobre as necessidades e expectativas relativas à atuação do psicólogo. Um levantamento realizado em unidades hospitalares, envolvendo observações do atendimento e entrevistas com os profissionais da saúde, subsidia as perspectivas adotadas pelos autores.

\section{MÉTODOSDEPREPARAÇÃOPSICOLÓGICA}

Preparação psicológica tem sido definida como uma estratégia planejada, utilizada por um psicólogo, ou por um auxiliar devidamente treinado, a fim de diminuir a ansiedade relacionada a procedimentos médicos, reduzir a dor, facilitar o processo de recuperação e tornar possível ao indivíduo lidar adequadamente com estes procedimentos. Freqüentemente empregadas de forma associada na prática hospitalar, é possível distinguir algumas principais categorias técnicas. Assim, as estratégias cognitivocomportamentais atuam tanto sobre os comportamentos que possam diretamente influenciar ou modificar as características objetivas de um evento ameaçador, como sobre o modo como o indivíduo interpreta, avalia e integra o evento. As mais comuns são: dessensibilização sistemática e autocontrole.Vale mencionar, também, o work of worrying, que inversamente à dessensibilização sistemática, visa sensibilizar o sujeito até um nível moderado de medo e/ou preocupação, com o objetivo de engajá-lo nos cuidados necessários (Reading, 1979).

As técnicas de modelagem envolvem, em geral, a aprendizagem de estratégias de enfrentamento adequado, seja através da observação de um modelo real, seja através de imagens como filmes, slides e figuras (Yap, 1988, Peterson, Schultheis, Ridley-Johnson, Miller \& Tracy, 1984).

As técnicas de suporte emocional e/ou expressivas privilegiam o papel das emoções no processo de enfrentamento da situação, introduzindo um agente de cuidados; em geral o psicólogo, que proporciona confiança, equilíbrio e segurança ao paciente, estabelecendo uma atitude de aceitação dos seus sentimentos e ansiedades, incentivando sua expressão. No caso de pacientes pediátricos, podem ser programadas atividades lúdicas livres ou estruturadas, com a manipulação de objetos que reproduzem as condições de tratamento, como por exemplo, seringas, bisturis, máscaras anestésicas (Yap, 1988; Brannon \& Feist, 1992).

O fornecimento de informações visa aumentar a tolerância ao estresse e diminuir a ansiedade, na medida em que aumenta a previsibilidade dos eventos, através do esclarecimento adequado e suficiente a respeito dos procedimentos médicos (Yap, 1988; Reading, 1979). A informação pode ser fornecida de diversas formas, dependendo dos recursos de que se dispõe e dos objetivos do trabalho: folhetos, audiovisuais, visitas de familiarização à instituição hospitalar, palestras. A natureza da informação pode ser genérica, esclarecendo apenas sobre alguns aspectos da rotina hospitalar, ou pode ser extremamente específica, descrevendo detalhes sobre a equipe, equipamentos e procedimentos (Araujo, Lins, Abreu, Ramos \& Almeida, 1996, Araujo \& Tubino, 1996).

As técnicas de relaxamento consistem no ensino de recursos de enfrentamento fundamentados na correlação entre afetividade e tônus muscular, de forma a proporcionar controle cognitivo e comportamental e reduzir a dor e o medo (Yap, 1988).

A eficácia comparativa dessas técnicas foi avaliada por Saile, Burgmeier e Schmidt (1988) ao realizarem uma meta-análise de 75 intervenções controladas, publicadas entre 1953 e 1985. Estes autores verificaram que apesar de amplamente difundidas, as técnicas de modelagem obtiveram escores abaixo da média. As técnicas baseadas em suporte emocional, mais onerosas do ponto de vista institucional, alcançaram resultados duas vezes superiores às de modelagem. Com importantes variações estatísticas, as técnicas de relaxamento e dessensibilização sistemática foram as melho- 
res avaliadas. Constataram, igualmente, que quanto mais invasivo e complexo é o procedimento médico, maiores são as probabilidades de sucesso da preparação.

\section{PRINCIPAIS VARIÁVEIS MODERADORAS}

Segundo Janis (1974), as variáveis envolvidas na preparação psicológica podem se situar em nível extrínseco, intrínseco ou relacional. O nível extrínseco corresponde à situação na qual o paciente se encontra e sobre a qual tem pouco controle devido à novidade $e$ ao desconhecimento deste tipo de experiência. O nível intrínseco inclui todas as variáveis específicas que caracterizam o paciente, como idade, sexo, estado físico e experiência prévia (Zuckerberg, 1994). O nível relacional resulta da conjugação dos outros dois níveis em associação com a atuação de terceiros (profissionais, acompanhantes, equipe e instituição).

Serão discutidas, a seguir, as principais variáveis moderadoras da preparação psicológica para procedimentos cirúrgicos e invasivos. É evidente que tais variáveis são fortemente interligadas na rotina hospitalar, no entanto, serão aqui introduzidas separadamente, visando fornecer maior clareza. Em razão da limitação do escopo do presente trabalho, não serão desenvolvidas as variáveis de ordem médica (tais como: especialidades cirúrgicas e modalidades de indução anestésica) e de ordem organizacional e institucional (rotina dos centros cirúrgicos, distribuição de tarefas, disponibilidade de recursos, planejamento ambiental, dentre outras).

\section{IDADE}

Segundo Melamed, Dearborn e Hermecz (1983), quando tomada simultaneamente ao tipo e ao tempo de intervenção preparatória a idade da criança pode ser considerada a principal variável determinante do sucesso do traba-
Iho preparatório. Vernon, Schulman e Foley (1966) afirmam que crianças na faixa etária de seis meses a quatro anos são mais suscetíveis de apresentar sintomas após serem submetidas a procedimentos invasivos, tais como: ansiedade de separação, perturbações do sono, distúrbios alimentares, agressividade contra autoridade e apatia. De forma análoga, Eckenhoff (1953) ressalta que crianças mais jovens são propensas a desenvolver mudanças de personalidade. Ora, a idade da criança vincula-se intrinsecamente a fase de desenvolvimento das suas relações com a mãe e outros adultos significativos. Assim, crianças mais velhas seriam mais diferenciadas, heterogêneas e integradas, possuindo mais recursos de enfrentamento face a situações de estresse, sobretudo para lidar com eventos ambientais desconhecidos, em comparação com crianças menores, que por sua vez seriam mais dependentes dos sinais comportamentais maternos (Knight et alii, 1979). Quanto a intervenção, alguns autores recomendam que para as crianças mais velhas, deve ser despendido mais tempo nafase preparatória, além de se estabelecer um intervalo entre o trabalho de preparação e o procedimento médico. Já para as crianças mais jovens, seria melhor realizar a preparação psicológica pouco antes da intervenção médica (Melamed, Dearborn \& Hermecz,1983; Peterson et alii,1984).

\section{SEXO}

Existem poucos dados disponíveis relacionando o sexo do paciente à preparação psicológica, visto que os autores limitam-se a caracterizar a amostra. Contudo, embora de forma ainda rudimentar, Shirley e Poyntz (1941) esboçam alguns aspectos diferenciais dos comportamentos emitidos por meninos e meninas durante procedimentos médicos. Segundo as conclusões deste trabalho, crianças do sexo masculino apresentam mais ansiedade quando em situações de estresse dentro do ambiente hos- 
pitalar, o que, segundo as autoras, é consistente com a teoria freudiana sobre o Complexo de Édipo, que pressupõe um medo de castração, extensivo às intervenções médicas. Aberastury (1972) acrescenta em relação ao tema de castração que a criança vivencia o processo cirúrgico como um esquartejamento de seu corpo, alterando a sua auto-imagem e seu esquema corporal, de tal forma que são suscitados sentimentos arcaicos ligados à fobia edípica (complexo de castração). O trabalho de Shirley e Poyntz (1941) afirma também que as meninas são mais maduras e aumentam suas habilidades fisiológicas e sociais mais rápida e progressivamente, ao passo que os meninos exibem maior dependência da mãe até atingir a puberdade.

\section{ESTILOS DE ENFRENTAMENTO}

A partir de medidas psicofisiológicas, Knight et alii (1979) analisam a relação inversa entre mecanismos de enfrentamento e a taxa de produção de cortisol em pacientes hospitalizados. Os dados coletados sugerem que certos tipos de defesas egóicas estão associadas a um enfrentamento saudável e efetivo. Eles concluem também que as crianças que utilizam defesas do tipo racionalização, ou intelectualização com isolamento, estruturadas de forma flexível, são mais hábeis em enfrentar de modo eficaz a situação hospitalar, do quê as crianças que utilizam os recursos da negação, da negação com isolamento, fuga ou projeção, estruturadas de forma rígida. Ainda sob o enfoque psicofisiológico, Harper, Tornatzky e Miczek (1996) afirmam que em indivíduos que se recuperam de intervenções cirúrgicas, e que tendem a ser mais estressados, há uma deterioração do ritmo circadiano, caracterizado pelo decréscimo dos índices de freqüência cardíaca e da temperatura corporal. De modo semelhante à Janis (1974), Shipley, Butte Horwitz (1979) também identificaram dois estilos de enfrentamento: o "reprimido", em que o indiví- duo demonstra tranqüilidade, mas as medidas fisiológicas, como freqüência cardíaca, indicam alto nível de ansiedade e o "sensível", em que as informações são solicitadas ansiosamente, mas, a medida em que são fornecidas, o paciente apresenta indicadores fisiológicos de tranqüilização.

\section{EXPERIÊNCIA PRÉVIA}

Estudos recentes mostraram que entre 35 e $60 \%$ de crianças que se submeteram a procedimentos cirúrgicos relatam dor, moderada ou intensa, mesmo com a administração de analgésicos. A tendência neste tipo de estudo é focalizar tal evento como uma conseqüência do estresse associado à situação de hospitalização e cirurgia (Palermo \& Drotar, 1996). O nível de ansiedade diante de uma situação de cirurgia estaria relacionado à experiência prévia da criança com uma situação semelhante. Neste caso, se as expectativas antecipatórias forem negativas, o indivíduo tenderá a comportar-se de modo a evitar os estímulos aversivos envolvidos nos procedimentos médicos (Melamed, Dearborn e Hermecz, 1983). Considerando a complexidade da experiência dolorosa, que é permeada por julgamentos subjetivos, Redelmeier e Kahneman (1996) sugerem que as lembranças dos pacientes a respeito de procedimentos médicos dolorosos podem influenciar suas decisões sobre tratamentos futuros.

\section{PRESENÇA PARENTAL}

A influência parental sobre as reações comportamentais de crianças a procedimentos médicos aversivos é um dos pontos mais discutidos nos estudos de preparação psicológica (Bösenberg, Williams, \& Reddy, 1996; Domingos, 1993; Tiedeman, 1997). Sabe-se que a presença dos pais influi sobre os comportamentos dos filhos na situação hospitalar, no 
entanto, não se sabe porque isso acontece, e quais os fatores que ocasionam as mudanças comportamentais observadas. Assim como Shaw e Routh (1982), Gross, Stern, Levin, Dale \& Wojnilower (1983) analisaram a reação de crianças em duas situações na presença e na ausência da mãe, tendo verificado que sujeitos mais jovens (de quatro a seis anos) emitiram mais choro, agressividade e resistência durante os procedimentos, notadamente quando suas mães estavam presentes. Cabe ressaltar que a presença materna afetou significativamente $o$ choro apresentado antes do procedimento invasivo, o que poderia ser entendido como um apelo à mãe para que retirasse a criança da situação aversiva. As vantagens da presença dos pais no centro cirúrgico, até a fase de indução anestésica, foram investigadas por Gauderer, Lorig e Eastwood (1989) e Araujo e Tubino (1996). Dentre as vantagens esperadas podem ser apontadas: diminuição das possíveis seqüelas psicológicas entre os pacientes, indução anestésica mais tranqüila e a diminuição da ansiedade dos pais. Praticamente todos os pais que participaram de ambos os estudos optaram por acompanhar os filhos. Posteriormente, o depoimento dos pais foi muito positivo. Jacobsen et alii (1990) correlacionaram o papel das expectativas parentais sobre a cooperação dos filhos com o comportamento efetivamente apresentado por estes durante um procedimento invasivo. Os resultados demonstraram forte correlação positiva, as quais podem ser atribuídas seja ao fato dos pais conhecerem bem o repertório comportamental de seus filhos conseguindo fazer predições acuradas, seja à influência de uma profecia auto-realizadora. Esta última hipótese corrobora conclusões de Lee e Greene (1969) a respeito da ansiedade parental como facilitadora da expressão emocional negativa das crianças. No entanto, estes autores remetem a origem dos distúrbios comportamentais apresentados pela criança à relação familiar prévia, anterior à hospitalização. Lollar, Smits e Patterson (1993) relacionam o estilo de percepção de dor da mãe como elemento que influencia a percepção de dor dos filhos.

\section{RELAÇÃO COM A EQUIPE DE SAÚDE}

Médicos e enfermeiros são considerados fontes de informação privilegiada sobre os procedimentos, o que destaca o papel da equipe de saúde como uma variável moderadora do processo de preparação psicológica, uma vez que exerce função determinante sobre o controle da ansiedade e expectativas do paciente, contribuindo para o esclarecimento de dúvidas, amenizando o medo e receio que são constantemente associados aos procedimentos médicos (Sheridan \& Radmacher, 1992; Ellerton \& Merriam, 1994). Evidentemente, o estresse e seus modos de enfrentamento pelas equipes cirúrgicas são relevantes para a compreensão e intervenção neste nível assistencial.

\section{ATUAÇÃO DO PSICÓLOGO EM CIRURGIA E PROCEDIMENTOS INVASIVOS}

O levantamento teve por objetivo descrever e caracterizar a atuação do psicólogo em unidades de saúde que realizam procedimentos cirúrgicos e/ou invasivos, em particular no que se refere à identificação e à adoção de métodos de preparação psicológica contemplados pela literatura especializada atual.

\section{METODOLOGIA}

O trabalho foi desenvolvido em quatro instituições hospitalares do Distrito Federal, nos seguintes serviços: Terapia Intensiva, Politraumatismo, Ginecologia e Obstetrícia, Cirurgia Plástica e Cirurgia Pediátrica. No total, foram realizadas entrevistas semi-estruturadas com 20 profissionais de diferentes áreas de intervenção em saúde: 8 médicos, 4 enfermei- 
ras, 2 auxiliares de enfermagem, 2 funcionárias administrativas, 3 psicólogas e 1 pedagoga. Para tanto, foi aplicado um roteiro de entrevista abarcando as seguintes categorias temáticas: descrição do serviço quanto à composição da equipe de saúde, objetivos assistenciais e fluxo de atendimento; identificação das funções e tarefas profissionais (com ênfase nos procedimentos cirúrgicos e invasivos); necessidades e dificuldades do funcionamento da unidade; descrição dos métodos de preparação psicológica adotados pelos diferentes profissionais; identificação de demanda de intervenção do profissional de psicologia; descrição, percepção e avaliação da atuação do psicólogo da unidade. Utilizaram-se, também, registros de observação cursivos diretos e indiretos, no intuito de descrever as tarefas assistenciais e, desta forma, complementar as informações obtidas nas entrevistas.

\section{RESULTADOS E DISCUSSÃO}

O levantamento revelou que em apenas um serviço (terapia intensiva), o profissional de psicologia não está efetivamente presente no organograma institucional. Entretanto, nas demais unidades estudadas, nas quais o psicólogo faz parte do quadro, verificou-se que as suas atribuições extrapolam o atendimento de um único setor. Vale dizer que o serviço de ginecologia e obstetrícia conta apenas com uma psicóloga, apesar da seu grande porte (96 leitos), enquanto em politraumatismo e na cirurgia plástica, inseridos em outra instituição hospitalar, intervém a mesma profissional. Além de indicar carência de recursos humanos, esta distribuição parece apontar pouca compreensão da intervenção psicológica do ponto de vista do planejamento organizacional. Ao optar pela designação desta categoria para ações pontuais, isoladas e insuficientemente programadas, abarcando mais de um setor, a instituição inviabiliza, sobremaneira, a realização de inter- venções tecnicamente recomendadas pela literatura especializada.

Se foi possível constatar o reconhecimento dos demais membros da equipe de saúde quanto à importância e necessidade das intervenções por parte do psicólogo, por outro lado, os relatos obtidos demostram uma percepção limitada do potencial de participação deste profissional, em particular no que se refere à aplicação de técnicas específicas. Nenhum procedimento desta natureza foi mencionado. Referências foram feitas sobretudo à necessidade de apoio à equipe, em razão de dificuldades de ordem individual, tais como: inabilidade para lidar com o estresse; despreparo para estabelecer relação terapêutica e orientar pacientes e familiares.

Dificuldades de ordem interpessoal e grupal também foram destacadas. Assim, o psicólogo seria apropriadamente percebido como o membro da equipe de saúde melhor habilitado para intervir na esfera das relações e comunicações, em decorrência da sua formação. No entanto, uma vez mais, Ihe são atribuídas funções que freqüentemente excedem as possibilidades de atuação do profissional lotado na unidade. Tais expectativas necessitariam da intervenção de um consultor externo para serem satisfeitas.

Parece preocupante o fato de que não foi possível claramente identificar nenhum método de preparação psicológica no discurso das três profissionais entrevistadas. Ao mesmo tempo, não se apresentou fundamentação teóricometodológica para as ações empreendidas. Afirmaram possuir formação psicanalítica, mas pouco articularam sobre a sustentação fornecida ao seu trabalho por tal abordagem. É importante destacar que a análise desenvolvida nesta investigação restringe-se a familiarização e aplicação de métodos de preparação psicológica em contexto específico. Não se pretende de forma alguma discutir outras esferas da intervenção psicológica, comentadas pelas entrevistadas. 
Enfim, foi possível evidenciar uma incongruência entre a atuação deste profissional e as necessidades das unidades focalizadas. No que diz respeito às expectativas da equipe de saúde, constata-se que surgem de modo pouco realístico, visto que envolvem demandas, cuja satisfação exigiria propostas de níveis distintos àqueles para os quais o psicólogo da unidade está habilitado ou em posição de atuar. Ao mesmo tempo, suas possibilidades são subestimadas, o que parece se agravar pelo aparente desconhecimento do próprio psicólogo.

É essencial, no entanto, lembrar que grande parte das medidas passíveis de serem adotadas requerem mudanças nos serviços de saúde, envolvendo custos e investimentos no plano pessoal, profissional, grupal e institucional, e que aqueles mais diretamente implicados nem sempre estão dispostos a arcar.

\section{CONSIDERAÇÕES FINAIS}

Conclui-se pela necessidade premente de treinamento e formação do psicólogo em métodos de preparação voltados para os pacientes, familiares e equipe em situação de cirurgia e procedimentos invasivos. É fundamental que se reconheça a relevância de tais ações para o controle da dor e reabilitação do indivíduo, dentro de um enfoque humanizado tanto para o paciente como para seu acompanhante e seu cuidador. Através da aplicação destas estratégias, devidamente elucidadas pelas investigações científicas, será possível elaborar propostas de cunho preventivo. No que tange ao interesse científico, sugere-se a realização de levantamentos semelhantes ao da presente investigação, tanto no intuito de identificar necessidades de formação, como de subsidiar o desenvolvimento de estudos de meta-análise no contexto brasileiro, a exemplo do que vem sendo feito em outros países.

\section{REFERÊNCIAS BIBLIOGRÁFICAS}

ABERASTURY, A Historia de una técnica: preparación psicoterapéutica en cirugía. Em Aberastury, $A(O r g)$, El psicoanálisis de niños y sus aplicaciones (p. 34-43). Buenos Aires: Paidós, 1972.

ARAUJO, T.C.C.F., LINS, M.P.B.E., ABREU, M.M.R., RAMOS, V.S.C. \& ALMEIDA,V.M.B. Manual de preparação psicológica da criança para o tratamento oncológico, Temas em Psico-oncologia, I Congresso Brasileiro de Psico-oncologia, São Paulo, 153-154, 1996.

ARAUJO, T.C.C.F. \& TUBINO, P.J.G. Efeitos da Participação Parental em Rotina de Centro Cirúrgico para a Adaptação Psicológica do Paciente Pediátrico. Psicologia: Reflexão e Crítica, 9 (2), 369-382, 1996.

BÖSENBERG, AT., WILLIAMS, G.D. \& REDDY, D. Attitudes towards parental presence at induction of anaesthesia. South African Medical Journal, 86 (6), 664-667, 1996.

BRANNON, L. \& FEIST, J. Health psychology an introduction to behavior and health. Belmont: Wadsworth Publishing, 1992.

DOMINGOS, N.A.M. Preparopara cirurgia: teste de programas psicológicos na redução de ansiedade de crianças e mães. Dissertação de Mestrado. Pontifícia Universidade Católica de Campinas. Campinas, 1993.

ECKENHOFF, J. E. Relationship of anesthesia to postoperative personnality changes in children American Journal of Diseases of Children, 86, 587- 591, 1953.

ELLERTON, M. \& MERRIAM, C. Preparing children and families psychologically for day surgery: an evaluation. Journal of Advanced Nursing, 19, 1057-1062, 1994.

FREUD, S. Au-delà du principe de plaisir. Em Essais de psychanalyse (p. 41-115). Paris: payot, 1981, 1920.

GAUDERER, M.W.L., LORIG, J.L. \& EASTWOOD, D.W. Is there a place for parents in the operating room? Journal of Pediatric Surgery, 24 (7), 705-707, 1989. 
GROSS, AM., STERN, R.M., LEVIN, R.B., DALE, J., WOJNILOWER, D.A. The effect of mother- hild separation on the behavior of children experiencing a diagnostic medical procedure. Journal of Consulting and Clinical Psychology, 51 (5), 783-785, 1983.

HARPER, D.G., TORNATZKY, W. \& MICZEK, K.A. Stress induced disorganization of circadian and ultradian rhythms: comparisons of effects of surgery and social stress. Physiology \& Behavior, 59 (3), 409-419, 1996.

JACOBSEN, P.B.,MANNE, S.L,GORFINKLE, K.,SCHORR, O.,RAPKIN, B. \& REDD, W.H. Analysis of child and parent behavior during painful medical procedures. Health Psychology, 9 (5), 559-576, 1990.

JANIS, I.L Psychological stress: psychoanalytic and behavioral studies of surgical patients. New York: Academic Press, 1974.

KNIGHT, R.B., ATKINS, A, EAGLE, C.J., EVANS, N., FINKELSTEIN, J.W., FUKUSHIMA, D., KATZ, J. \& WEINER, H. Psychological stress, ego defenses, and cortisol production in children hospitalized for elective surgery. Psychosomatic Medicine, 41 (1), 40-49, 1979.

LEE, J.S. \& GREENE, N.M. Parental presence and emotional state of children prior to surgery. Clinical Pediatrics, 8 (3),126-130,1969.

LOLLAR, D.J., SMITS, S.J. \& PATTERSON, D.L. Assesment of pediatric pain: an empirical perspectiva. Em Roberts, M.C., Koocher, G.P., Routh, D.K. \& Willis, D.J. (Orgs.) Readings in pediatric psychology (p. 247257). New York: Plenum Press, 1993.

MELAMED, B.G., DEARBORN, M. \& HERMECZ, D.A. Necessary considerations for surgery preparation: age and previous experience.Psychosomatic Medicine, 45(6), 517-525, 1983.
PALERMO, T.M. \& DROTAR, D. Prediction of children's postoperative pain: Ihe role of presurgical expectations and anticipatory emotions. Journal of Pediatric Psychology, 21 (5), 683-698, 1996.

PETERSON, L, SCHULTHEIS, K., RIDLEYJOHNSON, R., MILLER, D.J., \& TRACY, K. Comparison of three modeling procedures on the presurgical and postsurgical reactions of children. Behavior Terapy, 15, 197-203, 1984.

READING, A.E The short term effects of Psychological Preparation for Surgery. Science\& Medecine, 13, 641-654, 1979.

REDELMEIER, D.A \& KAHNEMAN, D. Patients' memories of painful medical treatments: realtime and retrospectiva evaluations of two minimally invasive procedures.Pain, 66, 3-8, 1996.

SAILE, H., BURGMEIER, R \& SCHMIDT, L.R. A meta-analysis of studies on psychological preparation of children facing medical procedures. Psychological and Health, 2, 107-132, 1988.

SHAW, E.G. \& ROUTH, D.K. Effect of mother presence on children's reation to aversive procedures. Journal of Pediatric Psychology, 7(1), 33-42,1982.

SHERIDAN, C.L. \& RADMACHER, S.A. Health psychology: challenging the biomedical modal. New York: John Wiley \& Sons, 1992.

SHIPLEY, R.H., BUTT, J.H. \& HORWITZ, E.A. Preparation to reexperience a stressful medical examination: effect of repetitious videotape exposure and coping style. Journal of Consulting and Clinical Psychology, 47 (3), 485-492, 1979.

SHIRLEY, M. \& POYNTZ, L. The influence of separation from the mother on children's emotional responses. Journal of Psychology, 12, 251-282, 1941. 
TIEDEMAN, M.E. Anxiety responses of parents during and after the hospitalization of their 5to 11-year-old children. Journal of Pediatric Nursing, 12 (2), 110-119, 1997.

VERNON, D.TA, SCHULMAN, J.L. \& FOLEY, J.M. Changes in children's behavior after hospitalization. American Journal of Diseases of Children, 111, 581-593, 1966.
YAP, J.N. Acritical directions review of pediatric preoperative preparation procedures: processes, outcomes, and future. Journal of Applied Developmental Psychology, 9, 359-389, 1988.

ZUCKERBERG, A.L. Perioperative approach to children. Pediatric Anesthesia, 41 (1), 15-29, 1994. 